\title{
¿Cuentan los filólogos españoles con una formación adecuada para el mercado de trabajo europeo actual?
}

\section{Rosario Frías Azcárate \\ Erica E. Fellinger Jusué}

Universidad de Alcalá. Departamento de Fundamentos de Economía e Historia Económica

Plaza de la Victoria, s/n. 28802 Alcalá de Henares

rosario.frias@uah.es

erica.fellinger@uah.es

\section{Resumen}

En este artículo, se presentan algunos de los resultados obtenidos en un estudio cualitativo sobre la trayectoria laboral de los licenciados en Filología en España.

La valoración global que realizan los filólogos de la carrera es que aporta unos contenidos que permiten acceder al mundo de la cultura y del saber. Sin embargo, su inserción en el mundo laboral resulta muy dificultosa.

Se indican los aspectos positivos y negativos que tiene el diseño de la carrera en relación con su integración en el mundo laboral contemplado en el espacio europeo.

Palabras clave: filólogos, formación, mercado de trabajo, inserción laboral.

Abstract. Do Spanish Philologists possess the adequate education for the European current labor market?

This paper offers some results from a study on the professional life of graduates in Philology in Spain.

Philologists' global assessment of their career is that it provides the content for access to culture and knowledge. However, their possibilities for obtaining a job are scarce both because of the lack of job opportunities for these graduates and the ill-designed curriculum for their professional life.

Finally, the negative and positive aspects in the curriculum design related to obtaining a job in Europe space are indicated.

Key words: philologists, training, labor market, access to work market.

\section{Sumario}

Introducción

Incidencia de la motivación y sus aspectos fundamentales a la hora de la elección de la carrera de Filología

Proceso formativo en la carrera de Filología: experiencia y valoración
Obtención de la licenciatura y el paso al mundo laboral

Conclusiones

Bibliografía 


\section{Introducción}

La inserción laboral de los titulados es algo sobre lo que se ha reflexionado e investigado de forma prolífera en los últimos años ${ }^{1}$, como consecuencia del compromiso adquirido por España para la adaptación de la enseñanza superior al espacio europeo basada en los Acuerdos de Bolonia de 1999 y del nuevo panorama laboral al que ha dado lugar la sociedad de la información y las tecnologías.

La configuración de esta nueva sociedad conlleva el surgimiento de nuevas formas de estructura ocupacional, caracterizada fundamentalmente por el surgimiento de nuevos puestos de trabajo, con una clara tendencia hacia el incremento de la ocupación «informacional» ${ }^{2}$ : ejecutivos, profesionales y técnicos.

La productividad proveniente de la innovación y la flexibilidad son factores esenciales en la situación actual y requiere de la adaptación curricular, incorporación y renovación permanente de información y conocimientos para un adecuado desarrollo laboral.

Por tanto, nos encontramos ante una situación caracterizada por el cambio (social, institucional, organizativo, educativo) que requiere adaptación de los perfiles de los profesionales a las nuevas formas de trabajo. Ya no se demandan enseñanzas para el desarrollo de tareas concretas, sino formación que favorezca la flexibilidad, incorporación permanente de información y conocimientos, movilidad y adaptación a las nuevas necesidades que se plantean en el mundo laboral.

Por otra parte, nos encontramos en un momento en el que es preciso adaptar la formación universitaria española al planteamiento de un espacio europeo de enseñanza superior (EEES) surgido en la Sorbona en 1998 y, posteriormente, desarrollado en Bolonia en 1999, con la adquisición de compromisos que se han ido fraguando y concretando en estrategias concretas desde Bolonia hasta Praga (2001), Salamanca (2001-2002), Barcelona (2002), Berlín (2003), etc.

Esta situación ha hecho necesario conocer el nivel de adecuación de la formación ofrecida por las universidades españolas, a partir de 1990, a los requerimientos del mundo laboral y el espacio europeo de enseñanza superior, con el fin de aproximar ambas realidades. Para la consecución de este objetivo, se han realizado diversas investigaciones en diferentes licenciaturas y diplomaturas. Se ha puesto mayor énfasis en el periodo inicial de esta etapa en las diplomaturas, por tratarse de estudios de primer grado y, por tanto, de los que hipotéticamente podían encontrarse más próximos a la adaptación a

1. Informe de inserción laboral, UMH, 2002; La inserción del universitario en el mercado de trabajo, Barcelona, 1996; Trayectoria laboral de los diplomados en biblioteconomía y documentación, Madrid, Universidad Complutense de Madrid, Consejo Social, 2000; Trayectoria ocupacional y laboral de los diplomados en estadística de la Universidad Complutense de Madrid, Madrid, Consejo Social, 1999; entre otros.

2. Castells, M. (1999). La era de la información. Madrid: Alianza. 
la nueva situación de la enseñanza superior (filología, enfermería, fisioterapia, óptica, etc.).

En este artículo, nos referiremos a la inserción laboral de los licenciados en Filología en sus diferentes ramas: clásica, hispánica, alemana, eslava, francesa, inglesa, italiana, románica, semítica, lingüística, árabe y hebrea y literatura.

Para conocer la inserción laboral de estos profesionales, se ha llevado a cabo un primer estudio de corte cualitativo mediante la realización de grupos de discusión, siguiendo la técnica desarrollada por Jesús Ibáñez en su obra clásica sobre el grupo de discusión ${ }^{3}$. Se han realizado cuatro grupos de entre siete y nueve componentes cada uno de ellos, de los cuales dos se constituyeron con varones y mujeres licenciados en 1998-1999. En uno de los grupos, sus componentes ocupaban puestos de trabajo relacionados con los estudios cursados en la licenciatura y, en el otro, tenían ocupaciones laborales que no estaban relacionadas con su titulación de filólogos. Los dos grupos restantes eran de varones y mujeres licenciados entre 1990 y 1998, en uno de ellos sus componentes se hallaban ocupando puestos de trabajo relacionados con la titulación de filólogo y, en el otro, eran desempleados o con ocupaciones laborales no relacionadas con la titulación. Por tanto, las variables de trabajo han sido: la pertenencia al plan de estudios anterior o posterior al curso 1993-1994, estar o no trabajando en algo relacionado con los estudios de Filología, el género y haber realizado otros estudios relacionados con la filología durante o después de la realización de la carrera.

La aplicación de esta técnica tiene como fin indagar, a través de las opiniones de los licenciados en Filología, sobre: a) las motivaciones y los mecanismos de acceso a la universidad, así como de la elección de la carrera; $b$ ) el itinerario seguido en la universidad para poder detectar los elementos fuertes y débiles de la cualificación profesional recibida y adquirida, y $c$ ) si durante el periodo de estudio de la carrera ha existido alguna relación contractual con el mercado de trabajo y, en su caso, si ello ha incidido en el desempeño laboral posterior. El tipo de información que se obtiene en esta clase de estudios podría dar acceso a una segunda fase de corte cuantitativo y permitiría que se produjera la idónea construcción de un cuestionario.

Aquí se presentan los resultados obtenidos en la investigación cualitativa, para poder obtener la información pertinente sobre la trayectoria ocupacional y laboral de los licenciados en Filología entre los años 1990 y 2000, en la que se han analizado los siguientes aspectos:

- La motivación para la elección de la carrera.

- El mecanismo de acceso a la universidad.

- El itinerario formativo seguido.

- La relación con el mundo laboral durante la realización de la carrera.

- El mercado de trabajo de los recién licenciados.

- La búsqueda del primer empleo. 


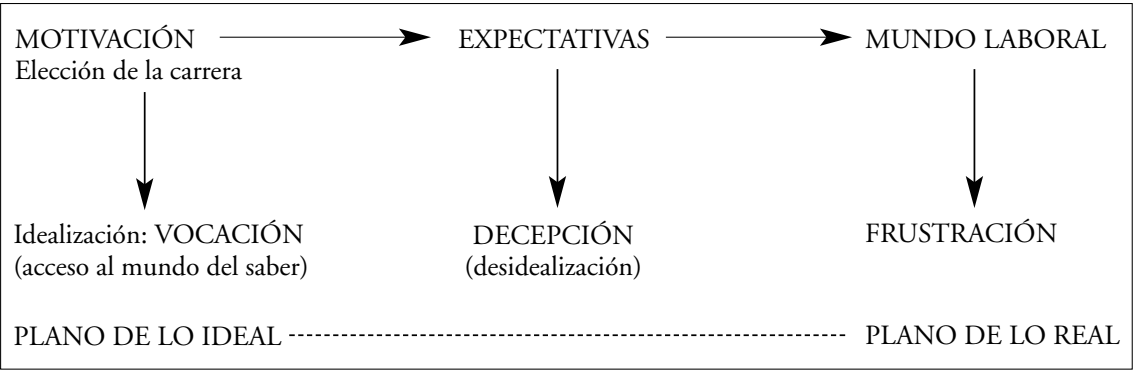

Figura 1. Tres momentos del proceso seguido por los licenciados en Filología.

La información de los resultados se ha estructurado en tres apartados que corresponden, cada uno de ellos, a los tres momentos más significativos del proceso seguido por los licenciados en Filología (figura 1):

- Los criterios fundamentales para la elección de la carrera.

- La experiencia y la valoración de la realización de la carrera de filología.

- La incorporación, tras finalizar la carrera, al mundo laboral.

El enfoque elegido para el análisis de los discursos producidos por los grupos ha sido el semiótico-estructural ${ }^{4}$ desde una perspectiva sociológica, atendiendo a la semiótica del relato y a los análisis estructurales de Lévi-Straus, Greimas y Barthes.

La producción discursiva de los grupos y también los ejes de análisis utilizados se han estructurado en función de los tres momentos anteriormente señalados.

Es necesario aclarar que los participantes en los grupos narran y valoran dichos momentos desde su situación actual, lo cual ha estado presente de forma específica en el análisis por dos razones: 1) porque toda percepción y valoración hecha desde el recuerdo desvirtúa en alguna medida la realidad de los hechos; 2) porque el análisis evidencia que existe una clara correlación entre los procesos cognitivo-valorativos sobre esos momentos y la situación laboral y profesional de los entrevistados en el momento presente.

Los discursos producidos por los grupos son bastante homogéneos en general; las diferencias surgen a la hora de realizar la percepción y la valoración de algunos temas, como, por ejemplo, la intensidad con la que se percibe la motivación, como elemento fundamental en la elección de la carrera, según se trate de unas u otras filologías.

En relación con las variables que han segmentado la muestra, las que han operado como discriminantes son: $a$ ) la pertenencia al plan de estudios anterior o posterior al curso 1993-1994, y b) que estén o no trabajando en algo relacionado con los estudios de Filología. 
De manera que: $a$ ) existe una clara correlación entre las personas que están realizando trabajos relacionados con la filología y, además, son las que mayoritariamente pertenecen al plan de estudios anterior al año 1993-1994, con una posición menos crítica sobre la carrera y las posibilidades profesionales que ofrece; $b$ ) los entrevistados que están en paro o que realizan trabajos que no tienen que ver con la filología, que coinciden mayoritariamente con los que pertenecen al plan de estudios posterior al curso 1993-1994, poseen un discurso más crítico.

El conjunto de los contenidos y las valoraciones que realizan sobre los temas tratados se hace desde posiciones muy críticas, tanto sobre la valoración de la carrera como sobre la situación laboral que ésta ofrece. El resultado de todo ello es que los aspectos negativos surgen siempre de manera espontánea, mientras que los aspectos positivos aparecen siempre de forma sugerida.

Las distintas filologías se han valorado en su conjunto, debido a que:

- Los objetivos que persigue el estudio toman como unidad de análisis la carrera de Filología en su globalidad.

- No existen diferencias importantes que adquieran significación específica entre unas y otras, excepto en el caso de los que han cursado Filología Inglesa, entre los que existe una cierta posición diferencial con el resto de las filologías.

\section{Incidencia de la motivación y sus aspectos fundamentales a la hora de la elección de la carrera de Filología}

La vocación se indica de forma espontánea como motivación fundamental a la hora de elegir la carrera de Filología.

Este aspecto vocacional adquiere una gran notoriedad, si bien opera con más intensidad en aquéllos que han estudiado Filología Clásica o Hispánica y decrece en los que han estudiado Filología Inglesa. Esto se debe a que en los primeros tiene una mayor presencia, como elemento de motivación, el mundo de la cultura, mientras que para los segundos el conocimiento del inglés no comporta sólo un interés cultural, sino también una posibilidad de obtener trabajo (figura 2).

Motivación Vocación: «El mundo de la cultura»

Filología $\left\{\begin{array}{l}\text { clásica } \\ \text { hispánica } \\ \mid \\ \text { inglesa }\end{array}\right.$

Figura 2. La vocación en los estudios de Filología. 
Este concepto de vocación pone en juego muchas de las ambivalencias y de las contradicciones que se desvelan tras el análisis de los discursos producidos por los grupos. Por ello, tiene una gran importancia en el conjunto de elementos significativos que estructuran la producción discursiva.

La vocación como motivación en la elección de la Filología que se pretende estudiar opera desde tres niveles:

- Orden del ser: se considera que para querer acceder a esta carrera hay que tener unas inquietudes previas que se relacionan con el deseo de adquisición de cultura y de «saber". Esto hace que se sientan diferentes a la mayoría, por tanto, se perciben como "minoría selecta», con intereses y valores que no son los dominantes en la sociedad actual. El sentido que adquiere este término se asocia con el origen etimológico del mismo, que se refiere a la idea de «llamada».

- Orden del tener: se piensa que la realización de esta carrera permitirá tener acceso al mundo de la cultura y del saber «con letras mayúsculas».

- Orden del sentir: al estar presente el deseo como motor motivacional, se evidencia que no sólo se trata de conseguir unos conocimientos sobre unas materias, sino de adentrarse en un proceso de «iniciación al mundo del saber», lo cual se afirma desde una intensidad emocional.

Por tanto, la vocación como elemento de motivación aparece desde una clara idealización de la carrera de Filología, pero también opera como justificación, ya que existe una necesidad de dotar de valor a la carrera desde el plano personal, ante la constatación de que ésta tiene escasa valoración, tanto laboral como social.

Esta posición de idealización genera unas expectativas que no se satisfacen en el proceso de realización de la carrera y, por tanto, se produce un sentimiento de decepción o frustración.

Un elemento que está implícito en la idea de vocación es la decisión de la elección de algo que se quiere. Ahora bien, manifiestan que dicha elección se hace por el gusto en sí mismo de estudiar Filología, sin intervenir en ésta la valoración sobre las posibilidades laborales futuras. Sin embargo, estas afirmaciones se hacen en gran parte desde un discurso del "deber ser». No se llevan a cabo sólo desde lo que se piensa y se siente, sino que éstas responden en cierta medida a lo que se supone que es lo correcto, que es lo que se debe decir.

En definitiva, al hablar de motivación, surge un discurso fuertemente racionalizador, discurso del «deber ser», que opera como elemento de legitimación de una decisión que, posteriormente, se traduce en insatisfacción por la frustración que produce el no ver cumplidas sus expectativas.

Asimismo, hay que destacar que lo vocacional está mucho más presente en las filologías hispánicas y clásicas, mientras que en Filología Inglesa tiene mayor peso aprender una lengua que puede servir como moneda de cambio en el mundo laboral, como herramienta fundamental para acceder al mercado de trabajo. El resto de las filologías estarían en una posición intermedia entre ambas (figura 3). 


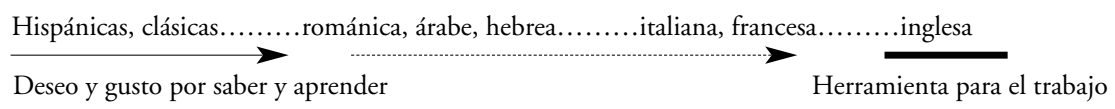

Figura 3. Valoración de las filologías.

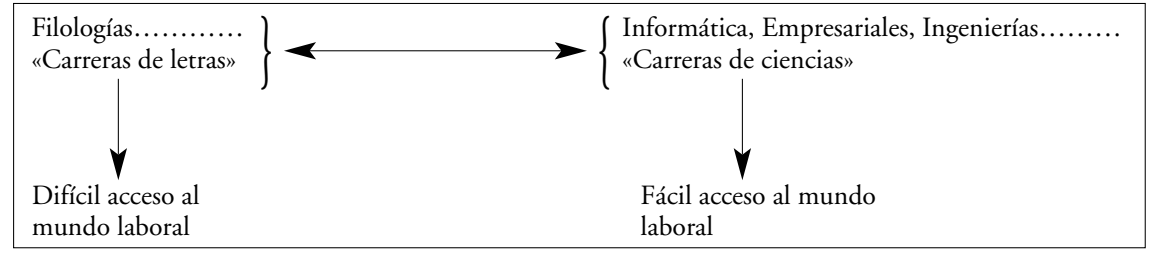

Figura 4. Valoración de la filología respecto a otras carreras.

Por último, es importante resaltar que la Filología se asocia de forma espontánea con las llamadas "carreras de letras", lo cual significa para los grupos que se trata de estudios que requieren unas inquietudes personales y culturales previas, que aportan un escaso potencial para la actividad laboral. En cambio, las llamadas "carreras de ciencias" son aquéllas que poseen un potente valor de cambio en el mundo laboral, sobre todo se mencionan las carreras de informática, empresariales e ingenierías (figura 4).

\section{Proceso formativo en la carrera de Filología: experiencia y valoración}

La valoración que los grupos realizan sobre su experiencia de la carrera viene condicionada por las expectativas previas que tienen sobre ella.

Como se ha indicado anteriormente, existe una idealización de los conocimientos que aporta la carrera de Filología. Este proceso de magnificación se relaciona con el mundo del saber, y los entrevistados establecen una identificación entre dicho mundo y la carrera. Por ello, esta situación trae como consecuencia inevitable que se sientan decepcionados, cuando no frustrados. La frustración es una de las razones que provoca una posición fuertemente crítica a la hora de valorar la carrera. Dicha posición es mantenida por la totalidad de los grupos, si bien algunas personas, especialmente las que trabajan en ámbitos relacionados con la filología y que pertenecen al plan de estudios anterior al curso 1993-1994, se sienten más bien decepcionadas, y esto hace que mantengan una postura crítica pero más racionalizada.

En relación con el tipo de filología estudiada, tampoco se producen diferencias significativas en su valoración, excepto la inglesa, sin olvidar que la diferencia se centra más en la «utilidad» final que en la carrera.

A continuación, se exponen esquemáticamente los elementos peor y mejor valorados para los entrevistados. 


\section{La carrera: aspectos más criticados}

— El plan de estudios «nuevo» (inicio del curso 1993-1994): excesivo número de asignaturas y horas de estancia en la facultad, falta de preparación y capacidad pedagógica del profesorado y dificultades para establecer relaciones entre los alumnos.

- Dificultad para encontrar utilidad de los conocimientos adquiridos, en el mundo del trabajo.

- Falta de información y asesoramiento para encauzar el currículo a la actividad laboral.

— Desorientación sobre la forma de acceder a la búsqueda de trabajo.

\section{La carrera: aspectos mejor valorados:}

- La experiencia del paso por la universidad.

- Los contenidos específicos de algunas asignaturas.

- La existencia de algunos profesores bien valorados por su formación, sus conocimientos y su capacidad pedagógica.

- Posibilidad de flexibilidad en la elección y organización de las asignaturas en el plan de estudios nuevo.

En definitiva, el análisis de los discursos pone de manifiesto que la percepción y la valoración que hacen los componentes de los grupos están atravesadas por un doble plano, que se refuerza y se retroalimenta: $a$ ) por un lado, está el plano de lo material, que tiene que ver con los aspectos más imbricados en la realidad, es decir, las asignaturas que han de cursar, la realización curricular que han de cumplir, los créditos que tienen que conseguir, etc., y $b$ ), por otro, está el mundo de representaciones simbólicas que se fijan a esos aspectos materiales, es decir, el mundo de las valoraciones sociales percibidas, de los referentes que adquieren o no prestigio social, que tienen una capacidad de poder simbólico (figura 5). Es la confluencia y la articulación de ambos niveles, pero especialmente el segundo, lo que hace que estemos ante unos discursos que, por un lado, critican a la carrera de Filología por las razones ya expuestas y, por otro, necesitan rescatar el sentido de los años y la dedicación a una disciplina que no saben cómo salvar. Desde aquí se rescata la vocación, el placer por la cultura, el gusto por algo, que muchas de las personas consideran a su vez «inútil».

\section{VALORES DE LOS ESTUDIOS DE FILOLOGÍA}

DESDE EL PLANO SIMBÓLICO

$(-)$ (no posee valor social)

(-) (escasa valoración académica)

(+) (valores desde lo personal): autoestima
DESDE EL PLANO FÁCTICO

(no hay valoración laboral)

- dificultad de acceso

- escaso reconocimiento profesional

- baja remuneración económica

Figura 5. Valores de la Filología. 


\section{Valoración global de la carrera}

En relación con la valoración global de la carrera, piensan que ésta tiene un enfoque generalista. Esto supone una posición de ambivalencia, ya que, por un lado, aporta una formación integral que permite una visión y una capacidad de análisis sobre los aspectos lingüísticos y literarios y, por otro lado, esta formación se valora como insuficientemente especializada, lo que hace que sientan que poseen poca capacitación para el trabajo.

\section{Obtención de la licenciatura y el paso al mundo laboral}

La finalización de la carrera y el inicio de la incorporación al mundo del trabajo representan un auténtico «rito de paso». Esto se traduce en:

- Afrontar una realidad que implica el acceso a la edad adulta y plantearse la integración en el mundo del trabajo.

- Detectar la utilidad que tienen los conocimientos adquiridos a la hora de buscar trabajo, así como contrastar el valor que tiene el título de Filología en el mundo laboral.

- Iniciar una nueva etapa formativa, que complementa la licenciatura aprendiendo y/o accediendo a títulos que se adapten a las exigencias del mundo del trabajo.

- Acceder de forma inevitable al mundo de lo real.

$\mathrm{Al}$ acabar la carrera, se encuentran con que algo que han elegido por la importancia que conceden al «acceder a la cultura», tiene un escaso reconocimiento en el mundo laboral, que se traduce en: pocas ofertas de trabajo, poca remuneración económica, escaso conocimiento de "para qué sirve un filólogo" y "qué sabe hacer un filólogo», excepción hecha de la enseñanza.

Contraponen la carrera de Filología con las carreras de ciencias e ingeniería, sobre todo se menciona Informática y Empresariales. Valoran estas carreras por sus mayores salidas laborales y por el reconocimiento laboral y social que poseen (figura 6).

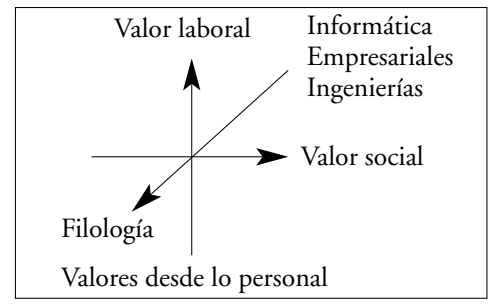

Figura 6. Valoración y posicionamiento respecto al resto de las carreras universitarias. 


\section{Trayectoria laboral de los filólogos}

En general, las personas que obtienen trabajo con más facilidad son las que pertenecen a la rama de lengua y, dentro de ésta, las que se inclinan por la Filología Inglesa. Ello se debe a la mayor oferta en este área en el ámbito de la enseñanza. Por el contrario, las personas que pertenecen a la rama de literatura son las que tienen más dificultad. Los trabajos que realizan mayoritariamente relacionados con su formación son:

- Docencia en la enseñanza privada: colegios y academias.

- Docencia en la enseñanza pública: concurso-oposición.

- Trabajo en empresas editoriales: lector, corrector y realizador de libros de texto.

\section{Conclusiones}

De la valoración global que se realiza sobre la carrera de Filología en sus aspectos fundamentales, se desprende que se trata de una disciplina que resulta atractiva porque aporta unos contenidos que permite acceder al conocimiento de la lengua y la literatura. En otras palabras, permite acceder al mundo de la cultura y del saber.

Sin embargo, el acceso al mundo laboral resulta muy dificultoso a causa de la oferta limitada de puestos de trabajo existente para los filólogos. Esto se debe a dos motivos fundamentales: la planificación académica mal diseñada para las competencias laborales, especialmente el plan de estudios posterior al curso 1993-1994, y el escaso reconocimiento, tanto social como laboral, que sufre esta titulación. Los licenciados de la rama de lengua y, dentro de ésta, los de Filología Inglesa son los que obtienen trabajo con mayor facilidad, debido a que existe más oferta en este área en el ámbito de la enseñanza.

En general, los filólogos contraponen su carrera con las de ciencias e ingeniería. Específicamente, las carreras de Informática y Empresariales son las más valoradas, a causa de sus mayores salidas de trabajo y el reconocimiento laboral y social que poseen.

El referente fundamental que opera como aspiración para los filólogos es la obtención de una plaza en la enseñanza pública. Esto es, porque además de acceder a un trabajo vinculado a los estudios realizados, supone una estabilidad de empleo y sueldo. Este último aspecto ha constituido un valor social en España que todavía no se ha erradicado, a pesar de que la situación actual ha cambiado profundamente y se encuentra determinada por valores tan diferentes como la flexibilidad y la movilidad laboral.

En los tres momentos analizados (elección de la carrera, realización de la misma e incorporación al mundo laboral tras su finalización), aparecen aspectos motivacionales claramente distintivos. La vocación constituye la motivación fundamental en la elección de la carrera. Durante su realización, existe una idealización de los conocimientos que se pueden adquirir, porque se establece una identificación entre el mundo del saber y la licenciatura. Por otro 
lado, la incorporación al mundo laboral representa un auténtico «rito de paso», ya que comienza una etapa formativa que complementa la licenciatura, cuya motivación es buscar conocimientos y obtener títulos que se adapten a las exigencias del mundo laboral.

La variedad de cursos complementarios realizados pone de manifiesto la necesidad de adquirir habilidades y competencias no transmitidas en su formación de grado. Lo filólogos reclaman que, en sus estudios de licenciatura, exista un equilibrio entre la formación generalista y la especializada, mayor adecuación del contenido de las asignaturas al mundo laboral y más información sobre el mismo.

Además, los egresados de Filología perciben una gran falta de información y asesoramiento que les permita conocer cómo acceder a las posibles vías profesionales. Este hecho advierte de la necesidad no sólo de información, sino también de una formación específica en materias de gestión empresarial, estudio de una lengua aplicada a los negocios y al entorno jurídico, nuevas tecnologías aplicadas a las lenguas, etc.

Por lo tanto, el diseño del plan de estudios ha de ser modificado, introduciendo en él nuevos criterios formativos conducentes a que el perfil del licenciado en Filología sea competitivo en el espacio europeo y, como consecuencia de ello, esté integrado en posiciones actualmente necesarias en este ámbito de flexibilidad y movilidad laboral.

\section{Bibliografía}

Albert Verdú, C. y otros (2000). «La transición de la escuela al mercado de trabajo en España: años noventa». Papeles de Economía Española, no 86, p. 42-58.

Alonso, L. E. (1998). La mirada cualitativa en sociología: una aproximación interpretativa. Madrid: Fundamentos.

A theorical and empirical analysis, with special reference to education. Nueva York: National Bureau of Economic Research, 1964.

BARTHES, R. (1971). Elementos de semiología. Madrid: Albero Corazón.

Brincones CAlVO, I. (1999). La Universidad de Alcalá en su entorno: formación y empleo. Madrid: Universidad de Alcalá.

Burón, J. (1984). Motivación y aprendizaje. Bilbao: Mensajero.

CASTELls, M. (1999). La era de la información. Madrid: Alianza.

Club GeSTIÓN DE CALIDAD (1998). Mejora en la formación universitaria: sugerencias desde la empresa. Madrid: Club Gestión de Calidad.

CONSEJO SOCIAL DE LA UNIVERSIDAD COMPLUTENSE DE MADRID (1999). Investigación sobre la trayectoria ocupacional y laboral de los diplomados en Estadística de la Universidad Complutense de Madrid. Madrid.

- (2001). Trayectorias laborales de los diplomados en Biblioteconomía y Documentación de la Universidad Complutense de Madrid. Madrid.

CURIER, J.; NEWSON, J. (1998). Universities and globalization: critical perspectives. California: Curier J. Newson J. Thousand.

Miguel, J. de y otros (2001). Excelencia. Calidad de las universidades españolas. Madrid: CIS.

Chomsky, N. (2000). On Misseducation. Nueva York: Rowman \& Litlefield. 
FERNÁNDEZ ABASCAL, E. G. y otros (2003). Emoción y motivación: La adaptación humana. Madrid: Centro Ramón Areces.

Fernández Enguita, M. (1993). «La profesión frente a su público». El País, 30 de marzo.

Frías AzCÁrate, R. y otros (1992). «Motivación o corazón adiestrado». Capital Humano, no 41, enero.

FRÍAS AZCÁRATE, R. (2000). «Una aproximación al concepto de comunicación y su consecuencia en la práctica de las instituciones». Nómadas. Revista Crítica en Ciencias Sociales y Jurídicas, $\mathrm{n}^{\circ} 1$. ISNN: 1578-6730, enero-junio.

García Ferrando, M.; LópeZ-Aranguren, E. (1991). «Experiencia de investigación social en la universidad española». Revista Española de Investigaciones Sociológicas, núm. 56. Madrid.

Guidens, A. (2000). Un mundo desbocado. Los efectos de la globalización en nuestras vidas. Madrid: Taurus ( $1^{a}$ ed. Londres 1999).

HARZBerg, F. (1980). Motivación. Bilbao: Deusto.

IBÁÑEZ, J. (1979). Más allá de la sociología. Grupo de discusión: técnica y crítica. Madrid: Siglo XXI.

LATIESA, M. (1990). La deserción universitaria. Madrid: CIS.

LÈVI-STRAUSS, C. (1977). Antropología estructural. Buenos Aires: Editorial Universitaria de Buenos Aires.

MANNING; Cullum-SWAN (1994). Narrative, content, and semiotic analysis. N. Y. Dezin \& Y. Lincoln (eds.).

Marcos Martín, F. A. (1994). Informática y humanidades. Madrid: Gredos.

MarTín, A. L. (1987). Motivación y satisfacción en el trabajo. Madrid: Aguilar.

MARTín GARCíA, V. (1999). «La evaluación universitaria: paso obligado de la calidad educativa». Sociedad y Utopía: Revista de Ciencias Sociales. Madrid.

PizArRo, N. (1979). «Metodología sociológica y teoría lingüística». Madrid: Ed. Alberto Corazón.

SÁNCHEZ, F. J. y otros (1986). Enseñanza universitaria y mercado de trabajo: el primer empleo de los titulados universitarios. Madrid: Fundación Universidad Empresa.

SÁNCHEZ MarTíneZ y otros (2003). Sociología de las profesiones. Pasado presente y futuro. Madrid: DM.

Servicio de Orientación y Planificación Profesional (SOPP) (2003). Inserción laboral del licenciado en documentación. Madrid: Universidad Carlos III.

- (2003). Inserción laboral de los licenciados en dirección y administración de empresas. Madrid: Universidad Carlos III.

SCHARGEL, F. P. (1997). Cómo transformar la educación a través de la gestión de la calidad total. Ibd, Limited. January.

Tena Artigas, J.; Cordero, L.; DíAz Jares, J. L. (1976). La universidad española: datos para un problema. Madrid: Confederación Española de Cajas de Ahorros. TODT, E. (1982). La motivación. Barcelona: Herder.

Val NúNEzZ, M. T. del (1997). Capacidad directiva de la empresa española: Potenciales de desarrollo de los directivos de recursos humanos en España. Ponencia. Congreso. 